DOI: https://doi.org/10.24297/jap.v16i1.8110

\title{
Synopsis and Review: Algebraic Quantum Theory of Consciousness by Elio Conte
}

\author{
Rich Norman \\ School of Advanced International Studies on Applied Theoretical \\ and Non Linear Methodologies of Physics, Bari, Italy. \\ Editor, Mind magazine; Editor, Journal of Unconscious Psychology; \\ Book Review
}

\section{Elio Conte}

Algebraic Quantum Theory of Consciousness

A Solution to the Problem of Quantum Collapse in Systems Having Three Anticommuting Elements

ISBN 978-88-255-1707-1, formato 17 x $24 \mathrm{~cm}, 112$ pagine, 14 euro

http://www.aracneeditrice.it/pdf/9788825517071.pdf

http://www.aracneeditrice.it/index.php/pubblicazione.html?item=9788825517071

Upon first reading the work of Conte some 6 years past, the impression was that of singular amazement. How could it be? The many rows of equations I puzzled through defined the mind itself, the mental transference creating perceived reality was represented in these many equations. How was it possible that the mind was so accurately described by these mathematics? In the upcoming years it became clear that quantum theory is actually a better descriptor of mental process than physical ones. The physical solutions derived by quantum theory are of the highest accuracy, but the causal element was neglected in physics [1]. However, within the sphere of mentation, we do see an accurate causal process description. This is due to the phenomenological constraints placed upon perceived reality, where the subject may never know of the outside world save by way of their subjective contextually defined internal impressions and memory. Indeed, the existential divide within mental processes is itself the exact defining factor, which connects the mind and quantum theory. In the mind, we always necessarily define reality from contextually defined potential, formed up into bound reality: the collapse of the wave function. This concept, is a mental one. There is good justification to the method within this book, which rightly defines the wave function collapse in a new and entirely quantum way, with no ad hoc postulates! In this bold move redefining wave function collapse algebraically, we approach the connectivity between mathematics of probability and mental functioning most closely.

The reason this mathematics succeeds so well in this subtle task is that it redefines the collapse as an entirely quantum encoding into bound reality, exactly as mentation truly does proceed. Mentation is not as the Schrodinger equation, it is not semi-classical, it is entirely probabilistic, fully quantum [2]. One need but observe the most primary and ancient brain anatomy, such as the PGO system and amygdala in adjusting orienting responses to understand, probability is the method the mind uses to bridge the phenomenological gap inherent in perception, and perceived vs. expected outcomes is the axis by which that probabilistically defined system is adjusted [2].

This tidy volume condenses Conte's major ideas into a neat and simple form for easy reference. The mathematics is somewhat daunting for many, so explanation was added in a chapter I have contributed to allow the reader a foothold to gain entry into the ideas. The genesis of quantum theory and its fundamental 
relation to mentation is covered, as well as more specific reference to the transference, spin theory and the neuroses. This is the mathematics not only of our physical world, but our minds as well.

The first mental act of the human which separates him from his environment, the act of selfhood and individuation itself, takes place within the first 18 months of life, and was presaged long ago in the ancestry of the human spanning back millions of years...self-corresponding to an ancient motor structure as defined by Panksepp [3]. Now Conte has given us mathematics to compliment our biology and evolutionary processes. As self becomes separate from the world, we have the basis of object representation, of object separation and hence counting: of mathematics. Self, is one object, the world, another. Once the biological organism has advanced in this way, probability comes to the fore to define all mentation. Within this fact we may then clearly see: biology and the mind are necessarily quantum, necessarily of probability. Now we have the mathematics of Conte, and may move a step on in defining and explaining this amazing fact--the fact of the quantum mind.

\section{References}

1. Norman, R.L.; Dunning-Davies, J. Probabilistic Mechanics: the hidden variable. In press: Hadronic Journal.

2.Norman, R., 2015, Quantum Unconscious Pre-Space: A Psychoanalytic Neuroscientific Analysis of the Cognitive Science of Elio Conte-The Hard Problem of Consciousness, New Approaches and Directions. Neuroquantology, 13, 487-501. ( http://dx.doi.org/10.14704/nq.2015.13.4.869)

3. Panksepp J. Affective Neuroscience: The Foundations of Human and Animal Emotions. New York, NY.: Oxford Press, 1998. 